\title{
Antibiotic combination therapy can select for broad-spectrum multidrug resistance in Pseudomonas aeruginosa
}

Vestergaard, Martin; Paulander, Wilhelm; Marvig, Rasmus L.; Clasen, Julie; Jochumsen, Nicholas; Molin, Søren; Jelsbak, Lars; Ingmer, Hanne; Folkesson, Sven Anders

Published in:

International Journal of Antimicrobial Agents

Link to article, DOI:

10.1016/j.jjantimicag.2015.09.014

Publication date:

2016

Document Version

Publisher's PDF, also known as Version of record

Link back to DTU Orbit

Citation (APA):

Vestergaard, M., Paulander, W., Marvig, R. L., Clasen, J., Jochumsen, N., Molin, S., Jelsbak, L., Ingmer, H., \& Folkesson, S. A. (2016). Antibiotic combination therapy can select for broad-spectrum multidrug resistance in Pseudomonas aeruginosa. International Journal of Antimicrobial Agents, 47, 48-55. https://doi.org/10.1016/j.ijantimicag.2015.09.014

\section{General rights}

Copyright and moral rights for the publications made accessible in the public portal are retained by the authors and/or other copyright owners and it is a condition of accessing publications that users recognise and abide by the legal requirements associated with these rights.

- Users may download and print one copy of any publication from the public portal for the purpose of private study or research.

- You may not further distribute the material or use it for any profit-making activity or commercial gain

- You may freely distribute the URL identifying the publication in the public portal 


\title{
Antibiotic combination therapy can select for broad-spectrum multidrug resistance in Pseudomonas aeruginosa
}

\author{
Martin Vestergaard ${ }^{\mathrm{a}, 1}$, Wilhelm Paulander ${ }^{\mathrm{a}, 1}$, Rasmus L. Marvig ${ }^{\mathrm{b}}$, Julie Clasen ${ }^{\mathrm{c}}$, \\ Nicholas Jochumsen ${ }^{\mathrm{d}}$, Søren Molin ${ }^{\mathrm{d}}$, Lars Jelsbak ${ }^{\mathrm{d}}$, Hanne Ingmer ${ }^{\mathrm{a}}$, Anders Folkesson ${ }^{\mathrm{c}, *}$ \\ a Department of Veterinary Disease Biology, University of Copenhagen, Copenhagen, Denmark \\ ${ }^{\mathrm{b}}$ Center for Genomic Medicine, Rigshospitalet, Copenhagen, Denmark \\ ${ }^{\mathrm{c}}$ National Veterinary Institute, Technical University of Denmark, Copenhagen, Denmark \\ d Department of Systems Biology, Technical University of Denmark, Copenhagen, Denmark
}

\section{A R T I C L E I N F O}

\section{Article history:}

Received 9 April 2015

Accepted 29 September 2015

\section{Keywords:}

Antibiotics

Combination therapy

Multidrug resistance

$\beta$-Lactams

Fluoroquinolones

Drug efflux

\begin{abstract}
A B S T R A C T
Combination therapy with several antibiotics is one strategy that has been applied in order to limit the spread of antimicrobial resistance. We compared the de novo evolution of resistance during combination therapy with the $\beta$-lactam ceftazidime and the fluoroquinolone ciprofloxacin with the resistance evolved after single-drug exposure. Combination therapy selected for mutants that displayed broad-spectrum resistance, and a major resistance mechanism was mutational inactivation of the repressor gene mex $R$ that regulates the multidrug efflux operon mexAB-oprM. Deregulation of this operon led to a broadspectrum resistance phenotype that decreased susceptibility to the combination of drugs applied during selection as well as to unrelated antibiotic classes. Mutants isolated after single-drug exposure displayed narrow-spectrum resistance and carried mutations in the MexCD-OprJ efflux pump regulator gene $n f x B$ conferring ciprofloxacin resistance, or in the gene encoding the non-essential penicillin-binding protein DacB conferring ceftazidime resistance. Reconstruction of resistance mutations by allelic replacement and in vitro fitness assays revealed that in contrast to single antibiotic use, combination therapy consistently selected for mutants with enhanced fitness expressing broad-spectrum resistance mechanisms.
\end{abstract}

(C) 2015 Elsevier B.V. and the International Society of Chemotherapy. All rights reserved.

\section{Introduction}

Combination therapy with two or more drugs is the standard treatment for infections with human immunodeficiency virus (HIV), Plasmodium falciparum, Mycobacterium tuberculosis and, in cystic fibrosis (CF) patients, Pseudomonas aeruginosa [1-4]. There are multiple rationales for combination therapy. The clinical considerations include therapeutically covering the spectrum of potential pathogens during polymicrobial infections or in acute infections for which the responsible micro-organism or resistance profile of the pathogen is unknown [5]. Using different classes of antimicrobial drugs in combination might result in synergistic antibiotic interactions that enhance the inhibitory effect [5]. None the less, the general benefits of combination therapy compared with single or sequential administration of antibiotics for treating bacterial infections have been difficult to conclusively demonstrate

\footnotetext{
* Corresponding author. Tel.: +45 35886311.

E-mail address: afol@vet.dtu.dk (A. Folkesson).

1 These two authors contributed equally to this work.
}

[4]. Combination treatment might even have negative effects in infections with Gram-negative bacteria owing to increased treatment costs and increased risk of adverse side effects. However, since combination therapy could potentially lower the rate of de novo antibiotic resistance evolution, it is considered a potential resistance management strategy [4].

Theoretically, the probability of mutations conferring resistance to two agents simultaneously is lower than the probability of mutations conferring resistance to a single agent. However, this probability applies only if resistance mutations occur independently and drugs are administered simultaneously for enhanced effect. Considering the pharmacodynamic and pharmacokinetic complexity of multidrug treatment, reaching synchronised, therapeutically inhibitory concentrations at the site of infection in the clinical setting is problematic [2,6]. General resistance phenotypes caused by biofilm growth or persister state, or the potential selection for broad-spectrum resistance mechanisms activated via one-step mutational events confounds predictions about the potential overall benefit of combination therapy over monotherapy as a tool for controlling the spread of antibiotic resistance. We propose the following model by which bacterial populations acquire 


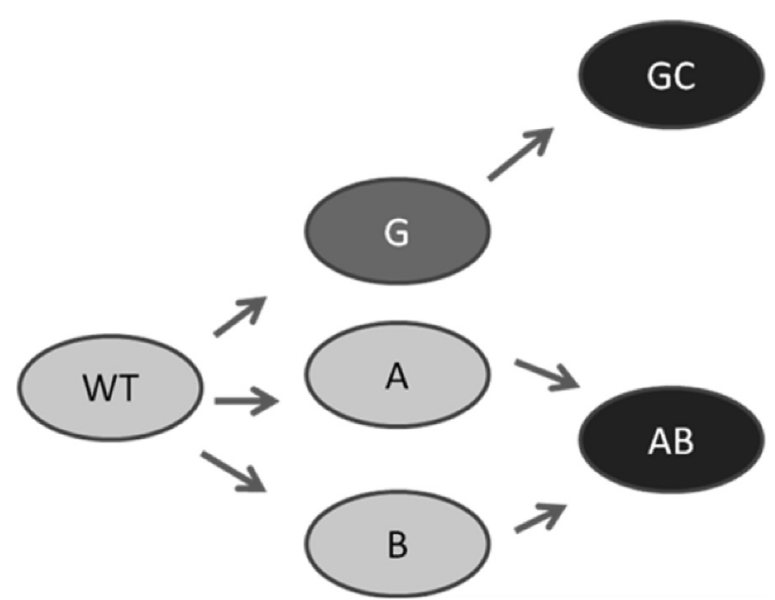

Fig. 1. Pathways for selecting general resistance mechanisms. A mutation or state that gives a general resistance phenotype has a selection advantage over specific resistance mutations when two drugs are present. ' $\mathrm{A}$ ' ( $\operatorname{dacB})$ and ' $\mathrm{B}$ ' ( $n f \times B)$ confer resistance to drug 'a' (ceftazidime) and drug 'b' (ciprofloxacin), respectively; ' $G$ ' (mexR or unknown) confers a general resistance phenotype; 'C' represents a compensatory event that either increases resistance or reduces the fitness cost of $\mathrm{G}$. The data from this study showed that $\mathrm{G}$ ( $\operatorname{mexR}$ or unknown) can occur at the same frequency as $A(d a c B)$ or $B(n f x B)$, increasing the probability of broad-spectrum resistance when two drugs are present. WT, wild-type.

broad-spectrum resistance during combination therapy. Resistance to antibiotics ' $a$ ' and ' $b$ ' is conferred by the resistance mutations ' $A$ ' and ' $B$ ', either through the acquisition of first $A$ and then $B$, or first $B$ and then A (Fig. 1). However, we hypothesise that in the presence of both drugs, a mutation or state that results in a general resistance phenotype (' $G$ ') towards a and b simultaneously confers a selective advantage compared with the specific resistance mutations ( $\mathrm{A}$ or $\mathrm{B}$ ) when the two drugs are present simultaneously. The $G$ state does not necessarily need to provide a survival advantage compared with an $A+B$ double mutant, but will have a fitness advantage compared with the A or B single mutant when both drugs are present. Moreover, the $G$ state will have the advantage of requiring fewer additional mutations to reach full resistance and will therefore change the resistance evolution trajectory by potentiating the evolution of novel resistance traits, either acquisition of the specific resistance mutations $a$ and $b$, which requires multiple mutations, or acquisition of an additional compensatory mutation ' $c$ ' that enhances $G$ to levels of resistance comparable with resistance provided by A and B (Fig. 1). To understand the consequences of antibiotic combination therapy, we investigated the spectrum of resistance mutations that were selected during single-drug, sequential drug and combination therapy. In vitro experiments were performed challenging the Gram-negative human pathogen $P$. aeruginosa with a $\beta$-lactam drug ceftazidime that targets cellwall synthesis, primarily penicillin-binding protein 3 (PBP3), and a fluoroquinolone ciprofloxacin that disrupts DNA replication by inhibiting the enzymes topoisomerase II and topoisomerase IV $[7,8]$. The antibiotics tested in the present study are used in the treatment of CF patients with $P$. aeruginosa lung infections, who are sometimes simultaneously treated with both drugs $[3,9]$. The molecular mechanisms underlying the resistance phenotypes were identified using whole-genome sequencing and allelic replacement. Each therapy selected for different resistance mechanisms, with combination therapy resulting in broad-spectrum resistance whereas antibiotic monotherapy selected for narrowspectrum resistance. These results have important implications for combination therapy as a clinical tool for antibiotic resistance management.

\section{Materials and methods}

\subsection{Strains, media, susceptibility testing and mutant selection}

Derivatives of $P$. aeruginosa strain PAO1 were used in this study [10]. A complete list of strains and plasmids is presented in Table 1. All strains were grown at $37^{\circ} \mathrm{C}$ in Luria-Bertani (LB) broth with aeration at $200 \mathrm{rpm}$ with or without ciprofloxacin (Bayer HealthCare AG, Leverkusen, Germany) and/or ceftazidime (Sigma-Aldrich, St Louis, MO). Minimum inhibitory concentrations (MICs) were determined for ceftazidime, ciprofloxacin and meropenem using Etest strips (bioMérieux S.A., Marcy-l'Étoile, France) on LB agar plates at $37^{\circ} \mathrm{C}$ for $22 \mathrm{~h}$ according to the manufacturer's guidelines. Etest MICs for combinations of ciprofloxacin and ceftazidime were determined according to the manufacturer's guidelines (Etest application sheet EAS 023; AB BIODISK, bioMérieux S.A.). Selection of resistant mutants was on LB agar plates containing antimicrobials at $5 \times$ MIC for PAO1 $(4 \mathrm{mg} / \mathrm{L}$ ceftazidime and $1 \mathrm{mg} / \mathrm{L}$ ciprofloxacin for singledrug therapy, and $1.5 \mathrm{mg} / \mathrm{L}$ ceftazidime $+0.2 \mathrm{mg} / \mathrm{L}$ ciprofloxacin for combination therapy). Single-drug selections were also performed at $1.5 \mathrm{mg} / \mathrm{L}$ ceftazidime and $0.2 \mathrm{mg} / \mathrm{L}$ ciprofloxacin. Mutation frequencies were calculated as antibiotic-resistant CFU divided by total CFU determined on antibiotic-free Luria agar plates.

Table 1

Additional strains used in this study.

\begin{tabular}{|c|c|c|c|}
\hline Strain & Relevant genotype/phenotype & Selecting agent $(\mathrm{g} / \mathrm{mL})$ & Source/reference \\
\hline PAO1 & Pseudomonas aeruginosa wild-type & & [10] \\
\hline MV146 & $\operatorname{mex} R(\mathrm{G} 188 \mathrm{~A})$ & & This study \\
\hline MV148 & $\operatorname{dacB}(\mathrm{G} 211 \mathrm{~A})$ & & This study \\
\hline MV176 & $\operatorname{dacB}(\mathrm{G} 211 \mathrm{~A})+n f \times B(\mathrm{C} 65 \mathrm{~T})$ & & This study \\
\hline MV182 & $n f x B(C 65 T)$ & & This study \\
\hline MV124 & & CIP 1 & This study \\
\hline MV125 & & CIP 1 & This study \\
\hline MV122 & & CAZ 4 & This study \\
\hline MV123 & & CAZ 4 & This study \\
\hline MV237 & & CIP $0.2+$ CAZ 1.5 & This study \\
\hline MV132 & & CIP $0.2+$ CAZ 1.5 & This study \\
\hline MV155 & & CIP $0.2+$ CAZ 1.5 & This study \\
\hline MV152 & & CIP $0.2+$ CAZ 1.5 & This study \\
\hline MV127 & & $\mathrm{CAZ} 4 \rightarrow \mathrm{CIP} 1$ & This study \\
\hline MV128 & & $\mathrm{CAZ} 4 \rightarrow \mathrm{CIP} 1$ & This study \\
\hline MV129 & & $\mathrm{CAZ} 4 \rightarrow \mathrm{CIP} 1$ & This study \\
\hline CC118 $\lambda \lambda$ pir & Escherichia coli lysogenised with $\lambda$ pir phage & & {$[11]$} \\
\hline pRK600 & RK2-Mob ${ }^{+}$RK2-Tra ${ }^{+}, \mathrm{Cm}^{\mathrm{r}}$ & & [12] \\
\hline pNJ1 & $s a c B$, Tet $^{r}$, R6 K origin & & {$[16]$} \\
\hline
\end{tabular}




\subsection{Genome sequencing and mutation analysis}

Genome sequencing of isolates was performed using an Illumina HiSeq $^{\mathrm{TM}} 2000$ machine (Illumina Inc., San Diego, CA) generating 100-bp paired-end reads as previously described [13] to an average genomic coverage of at least 74-fold (range 74- to 135-fold). Mutations were identified as previously described [14].

\subsection{Mutant construction}

Mutations from antibiotic-selected resistant strains were transferred by allelic replacement into PAO1 and triparental mating using the helper strain Escherichia coli CC118 $\lambda$ pir/pRK600 as described previously [15]. Primers for mutant construction are presented in Supplementary Table S1. Constructs with relevant gene products were incorporated into $\mathrm{pNJ} 1$ plasmid [16] and were used to transform the helper strain E. coli CC118 $\lambda$ pir. Selection of merodiploids was performed by plating the conjugation mixture on Pseudomonas isolation agar (Sigma-Aldrich) supplemented with $50 \mathrm{mg} / \mathrm{L}$ tetracycline as described previously [17]. Confirmation of incorporation of mutations was performed by PCR with mutationspecific primers [17].

\subsection{Quantitation of resistance gene expressions by quantitative $P C R(q P C R)$}

RNA was isolated using an SV RNeasy Mini Kit (QIAGEN, Hilden, Germany). RNA was converted to cDNA using a High-Capacity cDNA Reverse Transcription Kit (Applied Biosystems, Waltham, MA) with an RNase inhibitor. The cDNA was used as template for real-time qPCR using the primers in Supplementary Table S2 and Maxima SYBR Green/ROX qPCR Master Mix (Applied Biosystems). PCR products were detected using an MX3000P qPCR System (Agilent Technologies, Santa Clara, CA).

\subsection{Fitness measurements}

Three assays were used to estimate fitness. (i) The growth rate of bacteria grown in LB at $37^{\circ} \mathrm{C}$ with optical density at $600 \mathrm{~nm}$ measured over time using a Bioscreen $C$ reader (Labsystems Oy, Helsinki, Finland) and 100-well honeycomb plates with $300 \mu \mathrm{L} /$ well of overnight culture diluted to a starting concentration of $10^{6} \mathrm{CFU} / \mathrm{mL}$. The relative fitness of strains was calculated as the ratio of the mutant growth rate to that of the wild-type (WT). (ii) Stationary phase CFU were determined after $24 \mathrm{~h}$ of growth at $200 \mathrm{rpm}$ in $5 \mathrm{~mL}$ of LB medium in 50-mL polypropylene centrifuge tubes, with a starting inoculum of $\mathrm{ca} \cdot 10^{7} \mathrm{CFU} / \mathrm{mL}$. (iii) Competition experiments were used to determine relative competitive fitness (W) after $24 \mathrm{~h}$ for resistant strains isogenic to WT, which was calculated using the formula [18]: $W=\ln (\mathrm{RF} / \mathrm{RI}) / \ln (\mathrm{SF} / \mathrm{SI})$, where RI and SI are the CFU of resistant and susceptible cells at the start of the competition assay, respectively, and RF and SF are the number of resistant and susceptible cells at the endpoint of the competition assay. For the competition assay, overnight cultures of isogenic resistant and WT cells were diluted to ca. $10^{7} \mathrm{CFU} / \mathrm{mL}$, were mixed at 1:1 and were co-cultured for eight to nine generations before reaching stationary phase (using the same culturing conditions as for determining CFU described above).

Endpoint CFU (competition ratio) between resistant and WT cells was determined by spreading suitable dilutions on Luria agar plates with or without antibiotic concentrations appropriate for selecting the specific resistance. For each condition assayed, three to five biological replicates were used. For statistical analysis, one-way analysis of variance (ANOVA) with Dunnett's multiple comparison test as post-hoc analysis was used. All statistical calculations were performed using GraphPad Prism v.5.00 for Windows (GraphPad Software, San Diego, CA).

\subsection{Nucleotide sequence accession numbers}

Sequence reads from all isolates are deposited in the European Nucleotide Archive under study accession no. PRJEB8026.

\section{Results}

\subsection{High resistance frequency with the ciprofloxacin + ceftazidime antibiotic combination}

Combinations of antibiotics from four drug classes targeting distinct cellular functions were tested for synergistic effects against the reference strain $P$. aeruginosa PAO1 [10]. Ciprofloxacin was used in four combinations, paired with the third-generation cephalosporin ceftazidime, the carbapenems meropenem or imipenem, or the aminoglycoside tobramycin. Of the four combinations investigated, three showed an indifferent fractional inhibitory concentration index (FICI) using an Etest-based fractional inhibitory concentration assay [19]. Only the ceftazidime + ciprofloxacin combination had an additive effect $(\mathrm{FICI}=0.96)$. The ceftazidime + ciprofloxacin and ceftazidime + tobramycin combinations were selected for further investigations on resistance development. The resistance frequencies towards ciprofloxacin, ceftazidime and tobramycin were determined at $5 \times$ MIC for single, sequential and combination therapy. Owing to the additive effect from the tested antibiotic combinations, the absolute concentrations had to be adjusted in order to maintain a relative MIC exposure between the single and combination therapies. The fluctuation tests with single, sequential and combination therapy gave the following frequencies: monotherapy, $4 \times 10^{-8}$ for ciprofloxacin and $2 \times 10^{-7}$ for ceftazidime; sequential treatment, $5 \times 10^{-8}$ using both the single ciprofloxacin-resistant mutant (MV124) and the ceftazidimeresistant mutant (MV122) (Table 2). The resistance frequency of the ceftazidime + ciprofloxacin combination was $4 \times 10^{-8}$, showing that the selection for resistant mutants could neither be prevented nor substantially decreased with the combination of antibiotics. When the concentration was $10 \times$ MIC (ciprofloxacin $0.5 \mathrm{mg} / \mathrm{L}$ and ceftazidime $4 \mathrm{mg} / \mathrm{L}$ ) no mutants appeared. The ceftazidime + tobramycin combination could, however, prevent the emergence of resistant mutants at $5 \times$ MIC, therefore tobramycin monotherapy and sequential therapy were not further investigated.

\subsection{Combination therapy selects for broad-spectrum resistance mechanisms}

From each treatment procedure, a minimum of 20 independent resistant colonies were selected and resistance to ceftazidime and ciprofloxacin was investigated. Resistance to meropenem, which

\section{Table 2}

Frequencies of antibiotic resistance mutations for strains selected on CAZ, CIP, the $\mathrm{CAZ}+\mathrm{CIP}$ combination, or sequentially selected as $\mathrm{CAZ} \rightarrow \mathrm{CIP}$ or $\mathrm{CIP} \rightarrow \mathrm{CAZ}$.

\begin{tabular}{|c|c|c|c|}
\hline \multirow[t]{2}{*}{ Antimicrobial agent $\left(\mathrm{mg} / \mathrm{L}^{\mathrm{a}}\right)$} & \multicolumn{3}{|l|}{ Strain } \\
\hline & PAO1 & MV122 & MV124c \\
\hline CAZ 4 & $1.9 \times 10^{-7}$ & $\mathrm{~N} / \mathrm{D}$ & $5.3 \times 10^{-8}$ \\
\hline CIP 1 & $3.7 \times 10^{-8}$ & $4.7 \times 10^{-8}$ & $\mathrm{~N} / \mathrm{D}$ \\
\hline CAZ $1.5+$ CIP 0.2 & $3.9 \times 10^{-8}$ & $\mathrm{~N} / \mathrm{D}$ & $\mathrm{N} / \mathrm{D}$ \\
\hline
\end{tabular}

CAZ, ceftazidime; CIP, ciprofloxacin; N/D, not determined; MIC, minimum inhibitory concentration.

a Selection concentrations were $5 \times$ MIC of the sensitive PAO1 strain.

b CAZ-resistant strain.

c CIP-resistant strain. 
A

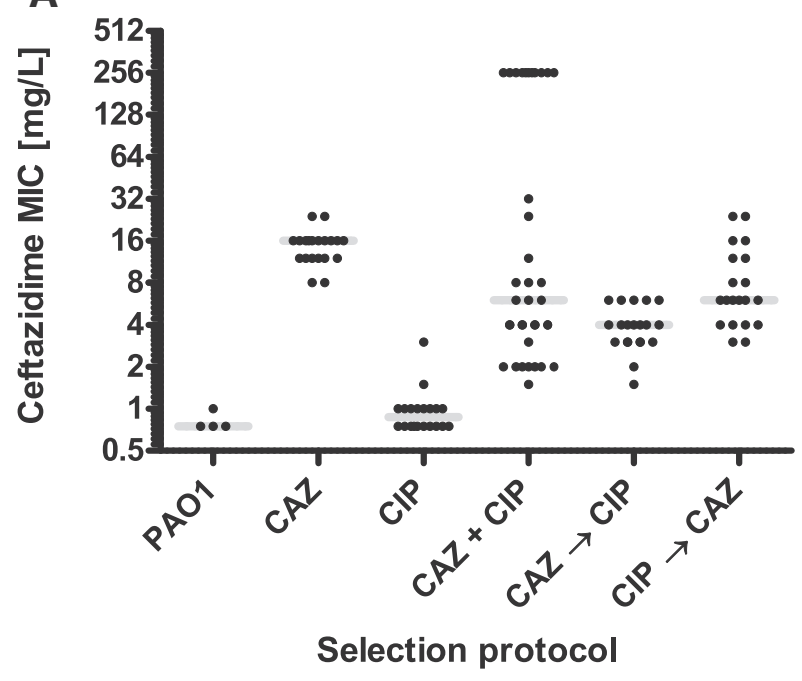

B

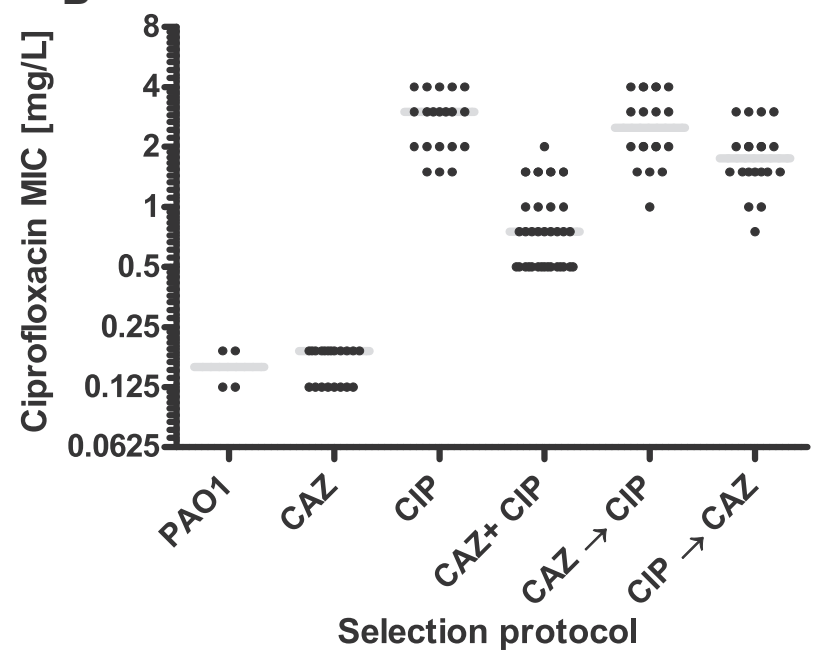

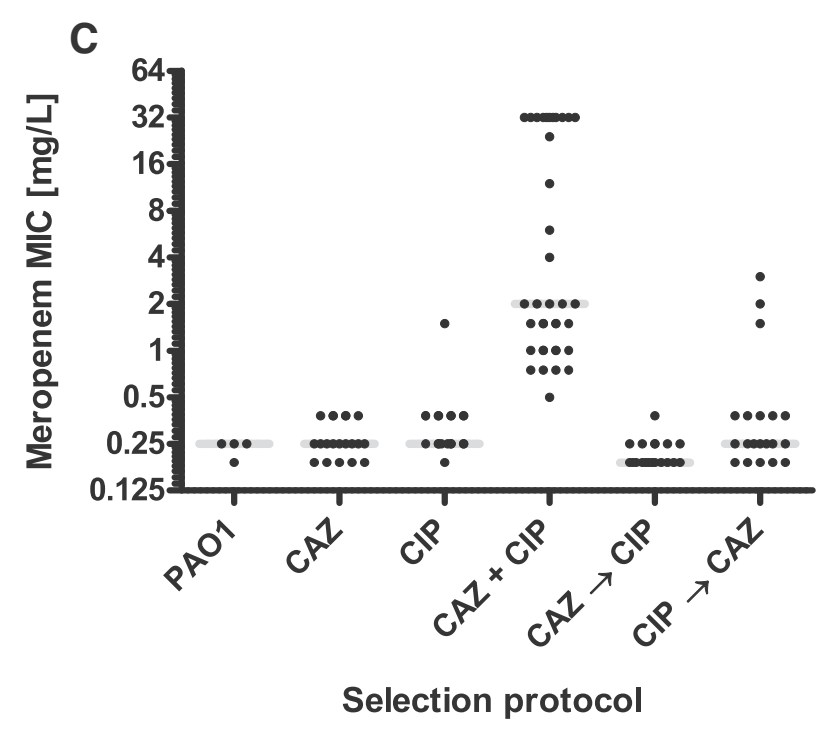

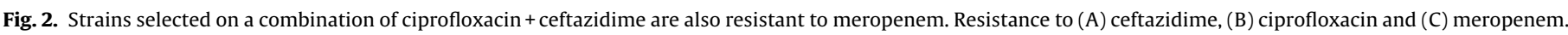

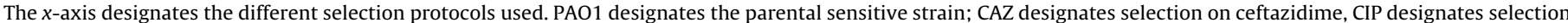

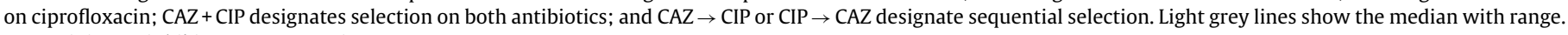
MIC, minimum inhibitory concentration.

was not used in any of the selections, was examined to assess broad-spectrum resistance (Fig. 2A-C; Supplementary Table S3). The median resistance of the ceftazidime-selected strains was increased 20-fold compared with the parental PAO1 strain (Fig. 2A). The ceftazidime-selected strains showed no changes in susceptibility to ciprofloxacin or meropenem (Fig. 2B and C) (Supplementary Table S3). The median MIC of ciprofloxacin-selected strains was increased 20-fold compared with the parental PAO1, and 19 of 20 ciprofloxacin-selected strains showed no change in resistance to ceftazidime or meropenem. Of 20 ciprofloxacin-selected strains, 1 had reduced susceptibility to both $\beta$-lactams (Fig. $2 \mathrm{~A}$ and $\mathrm{C}$ ) (Supplementary Table S3). However, all strains selected with the ceftazidime + ciprofloxacin combination displayed reduced susceptibility not only to ceftazidime and ciprofloxacin but also towards meropenem (Fig. 2A and C) (Supplementary Table S3). The median MIC for ceftazidime + ciprofloxacin-selected strains was $6 \mathrm{mg} / \mathrm{L}$ to ceftazidime and $0.75 \mathrm{mg} / \mathrm{L}$ to ciprofloxacin. The median susceptibility of ceftazidime + ciprofloxacin-selected strains to meropenem was 6-fold less than PAO1 (Fig. 2; Supplementary Table S3). Of 40 sequentially selected strains, 3 exhibited reduced susceptibility to meropenem, with resistance similar to ceftazidime + ciprofloxacin-selected strains; the other 37 strains exhibited decreased susceptibility only to ceftazidime and ciprofloxacin (Fig. 2; Supplementary Table S3).

To achieve a $5 \times$ MIC adjusted selection concentration for the combination and the single selection, the absolute concentrations of ciprofloxacin and ceftazidime were lower in the combination than for the single selections. Therefore, five independent mutants were selected on low-level ciprofloxacin concentration $(0.2 \mathrm{mg} / \mathrm{L})$ and ceftazidime $(1.5 \mathrm{mg} / \mathrm{L})$ corresponding to $1-2 \times$ MIC of PAO1.

Two of five strains ciprofloxacin-selected strains exhibited a general resistance phenotype with reduced susceptibility towards ciprofloxacin, ceftazidime and meropenem (Supplementary Table S3), whilst three strains showed specific resistance towards ciprofloxacin. Two ceftazidime-selected strains showed only resistance to ceftazidime, to similar levels as the strains selected on 
Table 3

Genome sequencing of resistant mutants selected on CAZ, CIP, the CAZ + CIP combination, or sequentially selected CAZ $\rightarrow$ CIP.

\begin{tabular}{|c|c|c|c|c|c|}
\hline \multirow[t]{2}{*}{ Strain } & \multirow[t]{2}{*}{ Selecting agent $(\mathrm{mg} / \mathrm{L})$} & \multicolumn{4}{|c|}{ Mutation } \\
\hline & & Gene & Type & Position $^{\mathrm{a}}$ & Amino acid change \\
\hline MV124 & CIP 1 & $n f x B$ & Insertion & $77(1 \mathrm{bp}) 78$ & \\
\hline MV125 & CIP 1 & $n f x B$ & Missense & C65T & P22L \\
\hline MV122 & CAZ 4 & $d a c B$ & Deletion & 1280(15 bp)1294 & \\
\hline MV123 & CAZ 4 & $d a c B$ & Missense & G211A & A71T \\
\hline MV237 & CIP $0.2+$ CAZ 1.5 & $\operatorname{mexR}$ & Deletion & $145(3 \mathrm{bp}) 147$ & \\
\hline MV132 & CIP $0.2+$ CAZ 1.5 & $\operatorname{mexR}$ & Insertion & $321(1 \mathrm{bp}) 322$ & Stop \\
\hline MV155 & CIP $0.2+$ CAZ 1.5 & $\operatorname{mexR}$ & Missense & G188A & $\mathrm{R} 63 \mathrm{H}$ \\
\hline \multirow[t]{2}{*}{ MV127 } & $\mathrm{CAZ} 4 \rightarrow \mathrm{CIP} 1$ & $n f x B$ & Deletion & 85(11 bp)95 & \\
\hline & & $d a c B$ & Deletion & $1280(15$ bp $) 1294$ & \\
\hline \multirow[t]{2}{*}{ MV128 } & $\mathrm{CAZ} 4 \rightarrow \mathrm{CIP} 1$ & $n f x B$ & Nonsense & C391T & Q131 \\
\hline & & $d a c B$ & Deletion & 1280(15 bp)1294 & \\
\hline \multirow[t]{2}{*}{ MV129 } & $\mathrm{CAZ} 4 \rightarrow \mathrm{CIP} 1$ & $n f x B$ & Missense & G541C & A181P \\
\hline & & $d a c B$ & Deletion & 1280(15 bp)1294 & \\
\hline
\end{tabular}

CAZ, ceftazidime; CIP, ciprofloxacin.

a Position of mutational change relative ATG start codon.

b MV122 used for sequential selection.

ceftazidime at $5 \times$ MIC (Supplementary Table S3), and three strains exhibited a general resistance phenotype with reduced susceptibility to ceftazidime, ciprofloxacin and meropenem.

\subsection{Mutations in mexR confer broad-spectrum resistance via derepression of MexAB-mediated efflux}

Whole-genome sequencing was performed on 10 selected strains to identify potential resistance mutations (Table 3 ). In all sequenced strains selected on ciprofloxacin, $n f x B$ mutations were identified. $\mathrm{NfxB}$ is a transcriptional regulator that represses expression of the mexCD-oprJ genes, which encode the drug efflux pump MexCD-OprJ. This is a common mechanism of resistance to ciprofloxacin in clinical isolates [20]. The two strains selected on ceftazidime had mutations in $\operatorname{dac} B$, which encodes the non-essential PBP4 that is involved in ceftazidime resistance through regulation of AmpC expression [21]. Mutations in mexR were identified as potential resistance-conferring mutations in the sequenced strains selected on ceftazidime + ciprofloxacin. The mexR gene encodes the transcriptional regulator MexR that represses expression of the mexAB-oprM genes encoding the efflux pump MexAB-OprM. Overexpression of this efflux pump is associated with broad-spectrum efflux of antimicrobial compounds in clinical Pseudomonas isolates [22]. Three isolates from a ceftazidime-resistant mutant (MV122) that was subsequently exposed to ciprofloxacin were sequenced. All contained the original mutation in $d a c B$ and had acquired additional mutations in $n f x B$ (Table 3). To confirm the effect of the mutations as the resistance mechanisms, allelic replacement was used to transfer mutations in $\operatorname{dacB}$ (G211A), $n f x B$ (C65T) and mexR (G188A) into the sensitive PAO1 background. No significant differences in resistance levels were observed between constructed and selected strains (Supplementary Table S3). The reconstructed mexR mutant had MIC levels above (ceftazidime) and at (ciprofloxacin) the drug concentrations used at the single-drug treatments. A strain with mutations in $d a c B$ (G211A) and $n f x B$ (C65T) was made to reconstruct the sequential acquisition of resistance mutations. The constructed $\operatorname{dac} B-n f x B$ double mutant showed resistance towards only ceftazidime and ciprofloxacin with no change in susceptibility to meropenem. The dacB-nfxB double mutant had resistance to ciprofloxacin (Supplementary Table S3) that was similar to the strain carrying only an $n f x B$ mutation but had a reduced MIC towards ceftazidime compared with the single $d a c B$ mutant.

\section{4. mexA expression is upregulated in most combination-selected mutants}

Mutations in the $d a c B$ gene influence $\beta$-lactam susceptibility by affecting expression of the native $\beta$-lactamase gene ampC [21]. Real-time reverse transcription qPCR was used to compare $a m p C$ expression in the reconstructed $d a c B$ strain (MV148) with PAO1 and 10 ceftazidime-selected strains. The mutation in $\operatorname{dac} B$ resulted in 110-fold upregulation of $a m p C$ mRNA in the reconstructed strain. Median expression of the ceftazidime-selected strains was 60-fold higher than the baseline PAO1 expression (Fig. 3; Supplementary Table S3). Ciprofloxacin-selected strains had a median increase in mexD expression of 443 -fold the PAO1 baseline (Fig. 3). The $d a c B-n f x B$ double mutant showed significantly $(P<0.01)$ lower $a m p C$ expression than the $d a c B$ single mutant. Expression of mexD was not significantly affected in the double mutant compared with the $n f x B$ mutant. Mutation in mexR increased mexA mRNA 11.6-fold (Supplementary Table S3) in the reconstructed strain compared with parental PAO1. Of 19 strains selected by ceftazidime + ciprofloxacin combination, 12 had a median 11-fold upregulated mexA expression (Fig. 3; Supplementary Table S3). Of the five strains selected at $0.2 \mathrm{mg} / \mathrm{L}$ ciprofloxacin, two showed

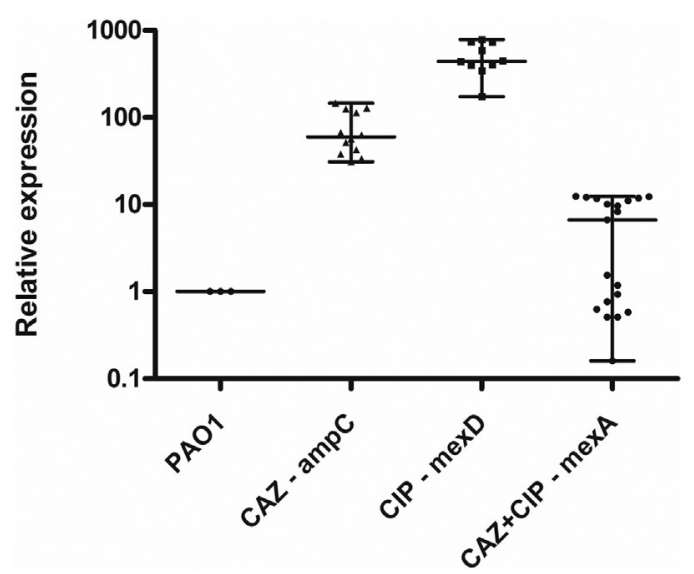

Fig. 3. mexA is upregulated in combination antibiotic-selected strains. Relative expression of relevant resistance mechanisms in bacterial isolates selected on CAZ $(\operatorname{amp} C), \mathrm{CIP}(\operatorname{mexD})$ or CAZ + CIP combination $(\operatorname{mexA})$. Quantification of gene expression was performed by quantitative PCR ( $\mathrm{PPCR}$ ) and was normalised to expression in the antibiotic-sensitive wild-type PAO1. Light grey lines show the median with range. CAZ, ceftazidime; CIP, ciprofloxacin. 
A

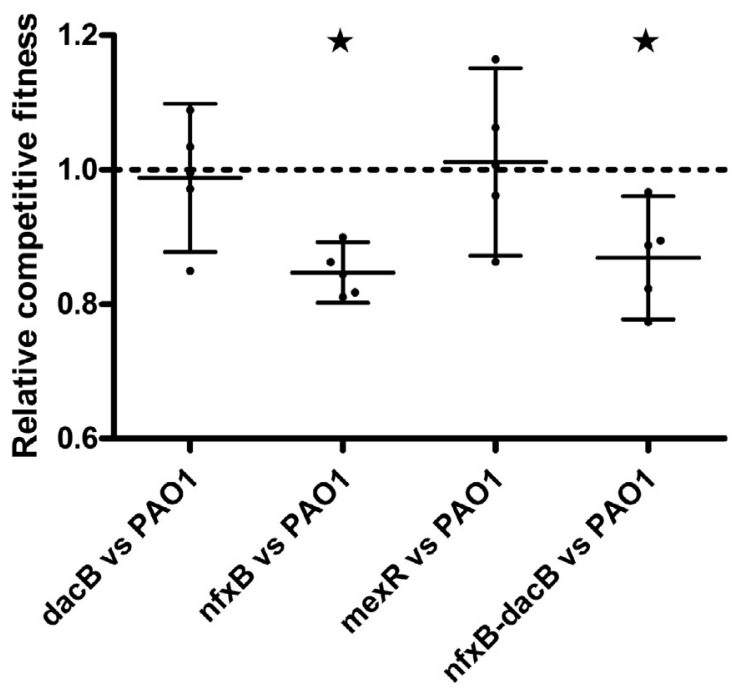

B

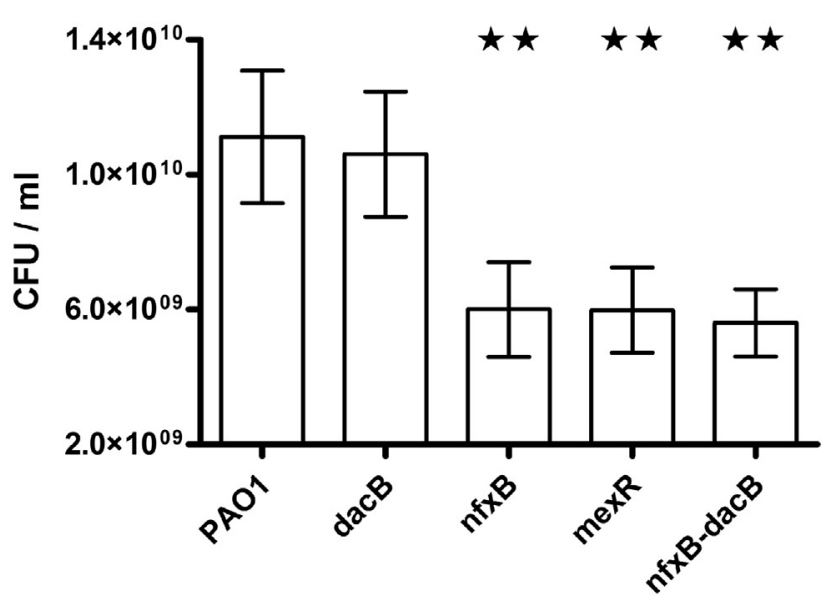

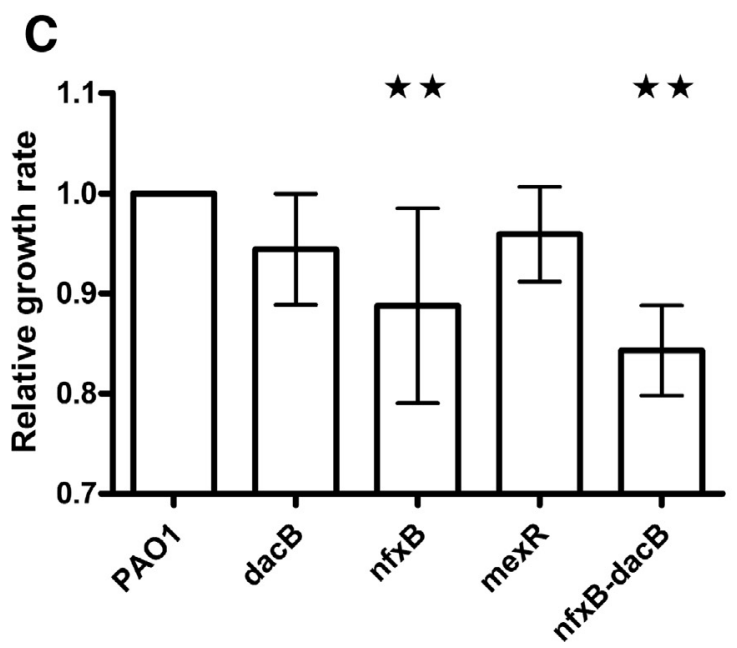

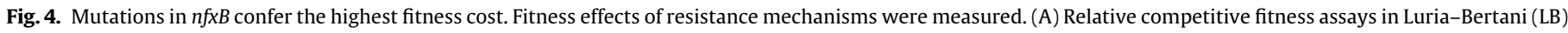

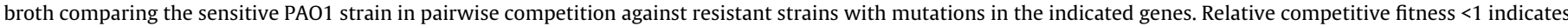

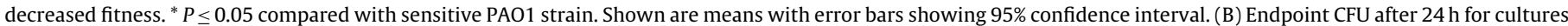

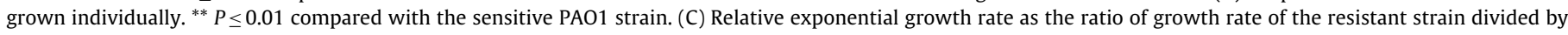
growth rate of the susceptible PAO1 strain. Growth rates were measured in LB and sensitive strains fitness was set to 1.0.

a 2.5-5-fold increased level of mexA expression. None of the remaining three had altered expression of mexD and the mechanisms of resistance in these strains remain unknown. Three of the five strains selected on $1.5 \mathrm{mg} / \mathrm{L}$ ceftazidime had increased expression of $\operatorname{ampC}$, whilst the resistance mechanism of the last two was not due to $\operatorname{ampC}$ overexpression.

\subsection{MexCD upregulation is associated with a competitive fitness cost}

To assess the potential fitness impact of the identified resistance mechanisms, the growth characteristics of the PAO1 strain was compared with four constructed isogenic resistant mutants ( $m e x R, n f x B, d a c B$ and $d a c B-n f x B$ ). The parameters analysed were: (i) exponential growth rate; (ii) CFU in stationary phase after $24 \mathrm{~h}$; and (iii) outcome of pairwise competitions between PAO1 and the four constructed mutants after inoculation at 1:1 and competitive growth for eight to nine generations (Fig. $4 \mathrm{~A}-\mathrm{C}$ ). In pairwise competitions in LB broth, a fitness cost was detected only for $n f x B$ and $d a c B-n f \times B$ mutants; no fitness cost was identified for $d a c B$ and mexR mutants (Fig. 4A). The relative competitive fitness of the $n f x B$ mutant to PAO1 was 0.85 . The fitness cost resulted from a combination of a reduced exponential growth rate of 0.89 relative to PAO1 and a two-fold reduction in CFU in a stationary phase culture. The fitness cost for the $d a c B-n f x B$ mutant was mainly associated with dysfunctional $\mathrm{NfxB}$ because the fitness for the three measured parameters were similar to the $n f x B$ mutant with relative competitive fitness of 0.87 , relative growth rate of 0.84 , and a two-fold reduction in CFU for a stationary phase culture after $24 \mathrm{~h}$ (Fig. 4A-C). The ceftazidime resistance mutation in $d a c B$ had no impact on relative competitive fitness (0.99) compared with PAO1 and no significant difference in growth rate or CFU count for a stationary-phase culture $(P>0.05)$. Similarly, the mexR mutant had a relative competitive fitness of 1.01 compared with PAO1 (Fig. 4A) and mexR mutants exhibited no significant reduction in exponential growth rate compared with PAO1 (Fig. 4B), but a two-fold reduction in CFU for a stationary-phase culture after $24 \mathrm{~h}$ was detected (Fig. 4C). 


\section{Discussion}

Determining the impact of combination therapy on antibiotic resistance evolution and bacterial fitness compared with the effect of monotherapy is important from both an evolutionary and a medical perspective. Combination therapy has been put forward as an alternative to decrease the rate of resistance evolution, but only a few studies have experimentally investigated combination therapy, and the data on the beneficial effects of combination therapy are conflicting [4]. We investigated the frequency of resistance and the spectrum of resistance mechanisms using ceftazidime, ciprofloxacin and tobramycin, three antibiotics commonly used to treat $P$. aeruginosa infections in CF patients. Two antibiotic combinations (ceftazidime + ciprofloxacin and ceftazidime + tobramycin) were assessed to determine changes in resistance evolution trajectories compared with single-agent usage or sequential exposure to the two agents. The ceftazidime + tobramycin combination prevented the selection of resistant mutants at the tested concentration, whilst resistant mutants were selected on the ceftazidime + ciprofloxacin combination. Therefore, resistance evolution to ceftazidime and ciprofloxacin during single, sequential and combination exposure was evaluated. In most resistant isolates, resistance after exposure to a single drug was attributable to increased expression of the cephalosporinase AmpC or the multidrug efflux pump MexCD-OprJ, indicating that mutations in the $d a c B$ and $n f x B$ regulators are responsible for the decreased antibiotic susceptibility (Fig. 2). Inactivating mutations in dacB are known to result in AmpC overproduction in clinical and in vitro-selected antibiotic-resistant $P$. aeruginosa isolates [21]. Ceftazidime and ciprofloxacin in combination resulted in resistant mutants at a frequency $\left(3.9 \times 10^{-8}\right)$ comparable with the frequency observed after exposure to ciprofloxacin alone (Table 2). In the majority of the strains, resistance was attributable to overexpression of the mexAB-oprM-encoding multidrug efflux operon, which facilitates the efflux of the two antibiotics [23]. Not all broad-spectrum resistant mutants exhibited mexA overexpression, indicating that at least one additional mechanism can confer broad-spectrum resistance. We were unsuccessful in identifying causative mechanisms in strains that were not overexpressing mexA.

We identified clinically relevant levels of resistance towards meropenem in strains isolated after exposure to a combination of ceftazidime and ciprofloxacin (Fig. 2A-C). Meropenem is a carbapenem belonging to a separate class of $\beta$-lactam antibiotics that is highly resistant to most $\beta$-lactamases [24]. The majority of the resistant strains isolated after exposure to a single drug had a resistance profile that was specific to the agent used for selection; only 1 of 40 investigated strains exhibited a broad resistance profile with decreased susceptibility to meropenem (Fig. 1C). The no-cost or low fitness cost associated with derepression of the MexAB efflux system (Fig. 4A-C) suggests that these mutants could both be pre-existing in a non-exposed population and will most likely stably remain in the population once the antibiotic pressure is removed $[25,26]$. The prevalence of isolates that overproduce an efflux pump in nosocomial $P$. aeruginosa strains is estimated to be $60 \%$, and as high as $75 \%$ in fluoroquinolone-resistant isolates [23]. Selection for broad-spectrum resistance mechanisms was not only identified during combination therapy, but also with antibiotic concentrations just above the MIC (1-2× MIC) the broad-spectrum mexA-related resistance could be identified (Supplementary Table S3), indicating large potential problems with dosing and suboptimal treatment regimens [27].

This work showed that treatment with combinations of antimicrobial agents has the potential risk of selecting for multiresistance mechanisms that confer broad-spectrum resistance. We demonstrate that the frequency of pre-existing mutations conferring resistance to two drugs simultaneously was not necessarily lower than the frequency of mutations after exposure to a single drug and that derepression of the MexAB efflux system is at little or no fitness cost. These results suggested that broad-spectrum resistance could emerge quickly during the use of some combination therapies in clinical settings. Interestingly, mutations in mexR and mexAB are among the genes most frequently associated with host adaptation in $P$. aeruginosa isolates from CF patients that have frequently undergone several different antibiotic combination therapies [3]. Moreover, recent in vitro investigations in E. coli indicate that combination therapy can preferentially select for efflux pump-mediated resistance [28]. In this work, combinations of several different classes of antibiotics preferentially selected for the multiple antibiotic resistance (mar) phenotype, indicating that the evolution of broad-range resistance may not only be restricted to ceftazidime + ciprofloxacin combination in $P$. aeruginosa [28]. In addition, extended resistance might be fixed in bacterial populations and difficult to eradicate once the resistance phenotype is present because of the low or non-existent fitness cost. The broad substrate range of many efflux mechanisms that confer resistance against drugs the bacterium was not previously exposed to is particularly worrying [29]. Our observation that multiple mechanisms conferring broad-spectrum antibiotic resistance could be selected with the ceftazidime + ciprofloxacin combination indicates that combination therapy does not necessarily lead to reduced resistance compared with single-drug exposure. We suggest that all antibiotic combinations should be analysed before being implemented in clinical settings (novel and already existing combinations) in order to assess the risk of selecting for broad-spectrum resistance mechanisms. These analyses are necessary in order to choose combination therapies that do not increase the risk of selecting for broad-spectrum resistance mechanisms.

\section{Appendix A. Supplementary data}

Supplementary data associated with this article can be found, in the online version, at http://dx.doi.org/10.1016/j.ijantimicag.2015. 09.014

\section{Funding}

This work was supported by the Danish Council for Independent Research [grants 274-08-0531 and 09-069656] and Ung Elitforskarpris [09-076146]. Bayer Schering Pharma is acknowledged for the generous gift of ciprofloxacin. The funders had no role in study design, data collection and analysis, decision to publish, or preparation of the manuscript.

\section{Competing interests}

None declared.

\section{Ethical approval}

Not required.

\section{References}

[1] Read AF, Day T, Huijben S. The evolution of drug resistance and the curious orthodoxy of aggressive chemotherapy. Proc Natl Acad Sci USA 2011;108(Suppl 2):10871-7

[2] Fischbach MA. Combination therapies for combating antimicrobial resistance. Curr Opin Microbiol 2011;14:519-23.

[3] Marvig RL, Sommer LM, Molin S, Johansen HK. Convergent evolution and adaptation of Pseudomonas aeruginosa within patients with cystic fibrosis. Nat Genet 2015;47:57-64. 
[4] Tamma PD, Cosgrove SE, Maragakis LL. Combination therapy for treatment of infections with Gram-negative bacteria. Clin Microbiol Rev 2012;25:450-70.

[5] Rybak MJ, McGrath BJ. Combination antimicrobial therapy for bacterial infections. Guidelines for the clinician. Drugs 1996:52:390-405.

[6] Cottarel G, Wierzbowski J. Combination drugs, an emerging option for antibacterial therapy. Trends Biotechnol 2007;25:547-55.

[7] Hayes MV, Orr DC. Mode of action of ceftazidime: affinity for the penicillinbinding proteins of Escherichia coli K12, Pseudomonas aeruginosa and Staphylococcus aureus. J Antimicrob Chemother 1983;12:119-26.

[8] Valerius NH, Koch C, Hoiby N. Prevention of chronic Pseudomonas aeruginosa colonisation in cystic fibrosis by early treatment. Lancet 1991:338:725-6.

[9] Diaz Caballero J, Clark ST, Coburn B, Zhang Y, Wang PW, Donaldson SL, et al. Selective sweeps and parallel pathoadaptation drive Pseudomonas aeruginosa evolution in the cystic fibrosis lung. MBio 2015;6:e00981-15.

[10] Stover CK, Pham XQ, Erwin AL, Mizoguchi SD, Warrener P, Hickey MJ, et al. Complete genome sequence of Pseudomonas aeruginosa PAO1, an opportunistic pathogen. Nature 2000;406:959-64.

[11] Herrero $M$, de Lorenzo V, Timmis $K N$. Transposon vectors containing non-antibiotic resistance selection markers for cloning and stable chromosomal insertion of foreign genes in Gram-negative bacteria. J Bacterio 1990;172:6557-67.

[12] Kessler B, de Lorenzo V, Timmis KN. A general system to integrate lacZ fusion into the chromosomes of Gram-negative eubacteria: regulation of the Pm promoter of the TOL plasmid studied with all controlling elements in monocopy. Mol Gen Genet 1992;233:293-301.

[13] Yang L, Jelsbak L, Marvig RL, Damkiaer S, Workman CT, Rau MH, et al. Evolutionary dynamics of bacteria in a human host environment. Proc Natl Acad Sci USA 2011;108:7481-6.

[14] Marvig RL, Johansen HK, Molin S, Jelsbak L. Genome analysis of a transmissible lineage of Pseudomonas aeruginosa reveals pathoadaptive mutations and distinct evolutionary paths of hypermutators. PLoS Genet 2013;9: e1003741.

[15] Choi KH, Schweizer HP. An improved method for rapid generation of unmarked Pseudomonas aeruginosa deletion mutants. BMC Microbiol 2005;5:30.

[16] Yang L, Hengzhuang W, Wu H, Damkiaer S, Jochumsen N, Song Z, et at. Polysaccharides serve as scaffold of biofilms formed by mucoid Pseudomonas aeruginosa. FEMS Immunol Med Microbiol 2012;65:366-76.
[17] Jochumsen N, LiuY, Molin S, Folkesson A. A Mig-14-like protein (PA5003) affects antimicrobial peptide recognition in Pseudomonas aeruginosa. Microbiology 2011;157:2647-57.

[18] Lenski RE, Rose MR, Simpson SC, Tadler SC. Long-term experimental evolution in Escherichia coli. I. Adaptation and divergence during 2000 generations. Am Nat 1991;138:1315-41.

[19] Pankey GA, Ashcraft DS. In vitro synergy of ciprofloxacin and gatifloxacin against ciprofloxacin-resistant Pseudomonas aeruginosa. Antimicrob Agents Chemother 2005;49:2959-64.

[20] Okazaki T, Iyobe S, Hashimoto H, Hirai K. Cloning and characterization of a DNA fragment that complements the $n f x B$ mutation in Pseudomonas aeruginosa PAO. FEMS Microbiol Lett 1991;63:31-5.

[21] Moya B, Dötsch A, Juan C, Blázquez J, Zamorano L, Haussler S, et al. $\beta$-Lactam resistance response triggered by inactivation of a nonessential penicillinbinding protein. PLoS Pathog 2009;5:e1000353.

[22] Poole K, Tetro K, Zhao Q, Neshat S, Heinrichs DE, Bianco N. Expression of the multidrug resistance operon mexA-mexB-oprM in Pseudomonas aeruginosa: $m e x R$ encodes a regulator of operon expression. Antimicrob Agents Chemother 1996;40:2021-8.

[23] Aeschlimann JR. The role of multidrug efflux pumps in the antibiotic resistance of Pseudomonas aeruginosa and other Gram-negative bacteria. Insights from the Society of Infectious Diseases Pharmacists. Pharmacotherapy 2003;23:916-24

[24] Livermore DM, Woodford N. Carbapenemases: a problem in waiting? Curr Opin Microbiol 2000;3:489-95.

[25] Andersson DI. Persistence of antibiotic resistant bacteria. Curr Opin Microbio $2003 ; 6: 452-6$

[26] Bjorkman J, Nagaev I, Berg OG, Hughes D, Andersson DI. Effects of environment on compensatory mutations to ameliorate costs of antibiotic resistance. Science 2000;287:1479-82.

[27] Gullberg E, Cao S, Berg OG, Ilback C, Sandegren L, Hughes D, et al. Selection of resistant bacteria at very low antibiotic concentrations. PLoS Pathog 2011:7:e1002158.

[28] Munck C, Gumpert HK, Wallin AI, Wang HH, Sommer MO. Prediction of resistance development against drug combinations by collateral responses to component drugs. Sci Transl Med 2014;6:262ra156.

[29] Poole K. Pseudomonas aeruginosa: resistance to the max. Front Microbiol 2011;2:65 